\title{
Index: Millennium-Studien zur Kultur und Geschichte des ersten Jahrtausends n. Chr.
}

\section{Orte und Namen}

Im Orts- und Namensverzeichnis werden die Ortsnamen in der jeweils aktuellen Landessprache geschrieben, historische Regionen und Personennamen unabhängig von der Sprache des Vorkommens in den Artikeln in ihrer deutschen Fassung. Abgekürzt werden Kaiser/emperor (Ks.), König/ king (Kg.), Königin/queen (Kgn.), Herzog/duke (Hz.), Graf/count (Gf.), Papst/pope (Pp.), Erzbischof/ archbishop (Eb.), Bischof/bishop (B.), Abt/abbot (A.).

Aachen 175, 177-178

Abbo von der Provence 16

Abbt, Christine 6

Ackermann, Josef 23

Adalbert 88

Ado 52

Adrevald von Fleury 89

Aega 55, 56

Aemila, B. von Mentesa 62

Aeneas 149

Aetius 100

Agapetus I., Pp. 125

Agaune 51, 55-56

Aggsbach 163

Agnes 180

Agobard 38

Agrestius $50-51,56$

Alarich II., Kg. $\quad 62,105,118$

Alcuin 98

Alethius 55

Alexander der Große 183

Alexander IV., Pp. 162

Alfons VI., Kg. 72

Alfons X., Kg. 73

Algazi, Gadi 30

Althoff, Gerd 16

Amandus 54, 57

Amatus 50, 51

Ambrosio de Morales 73-74

Anchises 149

Andernach 47

Angilram 149-150

Angoulême 87

Annegray 48

Ansbertus 150

Ansegisel 147-149

Aquitanien 82, 84-89
Arigius, B. von Lyon $\quad 46,59$

Aristoteles 98

Arles 116

Arnold von St. Emmeram 5, 6, 26-27

Arnulf 148-149

Arras 57, 58

Asser 37

Assmann, Aleida $\quad 4-5,17-18,190$

Assmann, Jan $\quad 4-5,17-20,36,190$

Astronomus 87-89

Attala, A. von Bobbio 49, 54

Athanasius, B. von Alexandria 9-11

Augustinus, B. von Hippo 2, 15, 92, 159, 182

Augustus, Ks. 185

Aurasius, B. von Toledo 76

Austrasien 52

Autharius 52

Babylon 183, 184

Bacon, Francis 5

Baldricus, Markgf. 87

Bamberg 181

Barcelona 179

Bartlett, Frederic C. 18, 20

Bayern, 23, 181

Beda Venerabilis $34,36-38,53,147$

Begga 149

Begos 88

Benedikt von Nursia 55

Benedikt von Aniane 87

Benedikt von Sant' Andrea 178

Benediktbeuern 24

Benjamin, Walter 61, 196

Bernhard von Clairvaux 25-26, 153, 156-158, 161

Bernhard von Chartres 4

Bernhard, Kg. von Italien 82 
Berta, Tochter Karls des Großen 84

Bertetrudis, Kgn. $\quad 55$

Bertram, B. von Bordeaux 140

Bertulf, A. von Bobbio 54, 56

Besançon 53

Bischoff, Bernhard 109-110

Blithild 149, 150

Bobbio 49, 54

Bonaventura 163

Boretius, Alfred 132

Borgolte, Michael 154

Bouchard, Constance 23

Bourges 88

Braulio, B. von Zaragoza 69

Bregenz 49, 52, 54

Bruges/Brugge 161

Brunhild, Kgn. $\quad 45,47,49,53,142$

Bruno von Köln 153-154, 160, 169

Bruxelles 161

Buccelin 44

Bulgar 76

Bully, Sébastien 48

Burgund 45, 50, 52

Caesarius, B. von Arles, 46, 48, 57, 118

Cambrai 58

Carmel 160

Carpetania 62

Carruthers, Mary 17, 25

Cartagena 62-64,68

Cassian 57

Cassiodor 9-11, 98

Cassirer, Ernst 196

Coelestin I., Pp. 128-129

Chagnerich 52-53

Chagnoald 52-53

Challet, Vincent 25, 26

Chalon-sur-Saône 46-56

Childebert I., Kg. $\quad 114,131,146$

Childebert II., Kg. $\quad 38,45,47$

Childebert III., Kg. 147

Childerich III., Kg. 141-142

Chilperich I., Kg. 140-141

Chlodwig I., Kg. 43, 114, 119, 133

Chlodwig III., Kg. 147

Chlothar II., Kg. $\quad 49,55,111,114,117,131-$ $133,140-141,143,148$

Chorso, Hz. von Toulouse 89

Chramnelenus 53

Chundo 47, 48
Chur 23

Cicero 5, 91, 98

Cîteaux 153

Clanchy, Michael 20

Clovis s. Chlodwig

Cluny 16, 155-158, 167

Coleman, Janet 25

Columban 42, 45-59

Comentiolus 63

Corbie 11

Cosmas von Prag 37

Froga, Gf. 76

Curta, Florin 35

Dado 52

Dagobert I., Kg. 56

Dagobert III., Kg. 147

Desiderius, Kg. $\quad$ 81-82

Devisse, Jean 134

Dhuoda 80-81

Didi-Huberman, Georges 195

Diem, Albrecht 51, 55

Dimbath, Oliver 6

Dinzelbacher, Peter 171

Doležalová, Lucie 25

Donatus, B. von Besançon 53, 55-56

Dondeyne, Albert 17

Drogo, B. von Metz 150

Dubischar, Markus 93

Duby, Georges 16

Duchesne, Louis 116, 125

Ebbinghaus, Hermann 18,

Eco, Umberto 6

Einhard 3, 81, 173-174, 178, 189-190, 193

Eligius, B. von Noyon 57-58

Elm, Kaspar 161

Elo 75

Erchempert 39

Erhart, Peter 23

Erlaldus 88

Ermoldus Nigellus $\quad 85-88$

Ervig, Kg. $\quad$ 70, 74-76

Eufemius, B. von Toledo 63

Eusebius, B. von Caesarea 94

Eustasius 49-55

Faremoutiers $\quad 52-54,56$

Fastrada 85

Felix III., Pp. 113, 130 
Felix IV., Pp. 125

Flavia 53

Flavius lohannis 43

Foucault, Michel 5, 197

Franziskus von Assisi 163,169

Fredegar 2, 36, 37, 41, 43, 55-56, 58, 70, 88, 101, 126, 194

Fredegunde, Kgn . 13, 140-141

Freising 22

Fridugis 86

Fried, Johannes 19, 30

Friedrich Barbarossa, Ks. 174, 177, 181

Friesland 179

Frutolf von Michelsberg 181, 185

Fruttuaria 155-158

Fulda 22, 23

Fulgentius 68

Gabriele, Matthew 177-178

Gallien $42-47,103,107,111,113,116,126-$ 127, 130, 177

Gallus 37

Garcia, Céline Frémaux 17

Geary, Patrick 1-11, 27, 29, 30-32, 38, 42, 56, 61, 137, 171, 187, 191-192, 195

Gelasius I., Pp. 113-114, 130

Gennadius von Marseille 69

Gerald von Salles 158

Gerhard, Gf. von Paris 88

Gerold, Markgf. 86

Gerung 86

Geuenich, Dieter 16

Gent 161

Gibitrudis 54

Gildas 46, 50

Gisela 84

Godomarus, Kg. 117

Goffart, Walter 33, 37

Goody, Jack 20

Gottfried von Viterbo 186

Gottschalk von Aachen 175

Gregor I., Pp. 36, 45, 125, 158

Gregor VII., Pp. 183

Gregor IX., Pp. 162

Gregor, B. von Langres 44

Gregor, B. von Tours 3, 37-47, 56, 58, 92 $104,128,140,151,193-194$

Grimoald 146

Große, Rolf 177

Gundemar, Kg. 61, 63-76, 193
Gundobad, Kg. $\quad 38$

Gundulf, B. von Metz 150

Gunthram, Kg. $\quad 44-48,56,99-100,131$, $140-141$

Hahn, Alois 101

Halbwachs, Maurice $\quad 4-5,18,28,190$

Harald Klaks, Kg. 86

Harries, Jill 127

Hautmont, Kloster 180

Heinrich IV., Ks. $\quad 175,180,183$

Heinzelman, Martin 103

Heito 82

Helisachar 86

Helladius, B. von Toledo 64

Henriquez, Chrysostomus 161

Heraclius, Ks. 71

Herodot 194

Hieronymus $\quad 69,94,147,183-184$

Hildegard, Kgn. $\quad 81,83$

Hildesheim 25

Hilduin 86

Hiltrud 84,85

Himerius, B. von Taragona 129

Hinkmar, Eb. von Reims 89-90,111, 134

Hirsau 158

Hobbit 28

Honorius I., Pp. 54

Horaz 108

Hormisdas, Pp. 125

Hrabanus Maurus 22

Hugo, Gf. von Tours 86

Humbert, Gf. 88

Hummer, Hans 21, 137

Hunna 56

Ibas, B. von Edessa 51

Ildefonsus, B. von Toledo 69, 76

Immo, Gf. von Périgueux (?) 88

Immonen, Teemu 24

Ingelheim 19

Innozenz I., Pp. 128

Innocentius, B. von Merida 62

Iohannis Scytha 43

Irmgard, Ksn. 82

Isidor, B. von Sevilla 37, 61-74, 98, 193

Iulius Victor 98

Johannes von Salisbury, B. von Chartres 186

Johannes II., Pp. 118 
Johannes von Réôme, A. von Moûtier-Saint-en-

Auxois $44-45,56$

Jonas von Bobbio 41-58, 194

Jordanes 34, 36-39

Judith, Ksn. 83

Julian, B. von Toledo $70,74-76$

Julius Cäsar 184

Julius I., Pp. $\quad 9-11$

Justinian, Ks. 51

Karl der Große, Ks. $\quad 23-24,81-90,143$, 173-179, 189, 194

Karl der Kahle, Kg. $\quad 80,90,178$

Karl Martell 146-147

Karlmann, Kg. $\quad 89,147$

Köln 153

Konrad III., Kg. 181, 184

Krusch, Bruno 42-43, 57, 144

Laon 82

Leander, B. von Sevilla 63,68

Leo I., Pp. 128

Leo III., Pp. $\quad 84,175,177$

Leo, B. von Civita Vecchia 86

Leopold III., Markgf. 180

Lérins 44,57

Leubardinus, A. von Moutiers St. Jean 44

Leudemund, B. von Sitten 55

Lévi-Strauss, Claude 29

Levillain, Léon 177

Liberius 117

Licinianus, B. von Cartagena $\quad 63,68$

Liutgard, Kgn. 84

Liuthard, Gf. von Fézensaz 88

Lorsch 23

Lothar I., Ks. 85

Lucas, B. von Tuy 72

Ludwig der Deutsche, Kg. 85

Ludwig der Fromme, Ks. $\quad 82-88,90,144,179$

Luxeuil 45, 47-50, 52-58

Lyon 47,115

Maassen, Friedrich $\quad 108,115-116$

Mâcon 46, 50

Magister Vincentius 37

Magnarius 88

Marcellus von Ancyra 9

Marchiennes 56

Marius, B. von Avenches 43, 44

Martianus Capella 98
Massona, B. von Mérida 63

Matfrid, Gf. von Orléans 86

Mathisen, Ralph 115-117

McKitterick, Rosamond 19, 20, 25

Meaux 52

Meerman, Gerard 107

Mérida $\quad 62,68$

Merleau-Ponty, Maurice 17

Metz 150

Meuthen, Erich 177

Mietius, B. von Langres 49

Miguel de Luna 73, 74

Mommsen, Theodor 108, 132

Mondsee 22

Monte Cassino 24, 25

Moore, Robert lan 171

Mordek, Hubert $\quad 46,108,115$

Morimond 180

Morris, Colin 172

Mummolus, Hz. $\quad$ 43-44

Mummolus, B. von Langres 44

Namer, Gérard 28

Nantes 52

Narbonne 88

Nicaea 10,119

Nicetius, B. von Trier 43

Nietzsche, Friedrich 5

Nora, Pierre 4, 5, 17-18, 28, 29, 190

Novalesa 24

Oexle, Otto Gerhard 5, 16, 150

Origenes von Alexandria 95

Orléans $100,116,119$

Orosius $38,182-183,185$

Otto, B. von Freising 180-186

Pappolus, B. von Langres 44

Paris 52, 57

Paschasius Radbertus, A. von Corbie $\quad 81,82$

Passau 22

Paulinus, B. von Aquileia 85

Paulus Diaconus 34, 36, 38-39, 101, 149

Pelagius I., Pp. 129

Périgueux 88

Petrus Venerabilis 167

Philipp I., Kg. 177

Phillipps, Thomas 107, 132

Phocas, Ks. 71

Pippin der Bucklige 89 
Pippin I., Kg. $\quad$ 141-143, 147, 148

Pippin II., Hausmeier 142, 149

Pippin von Aquitanien $84-86,88$

Pippin von Herstal 89

Plectrudis 89

Pohl, Walter $25,139,188$

Poitiers 88

Prinz, Friedrich 51

Probus, B. von Tortona 54

Procop 43, 44

Pseudo-Turpin 179

Pseudoisidor 10,11, 153

Raho, Gf. von Orléans 89

Rainald von Dassel, Eb. von Köln 181

Ranke, Leopold von 28, 188

Ravenna 195

Rekkared, Kg. $\quad 63,70$

Regensburg 5, 22, 27

Regino von Prüm 174

Reginpert, B. von Limoges 88

Reimitz, Helmut 25, 32-33, 37, 103

Reims 43, 107, 134

Remedius, B. von Chur 23, 24

Remiremont 51-52, 55

Renatus Profuturus Frigiretus 95

Rhodhaid 84

Rhodrud 84

Ricoeur, Paul 100

Robert, A. von Molesme 153, 160

Roderich, Gf. 83

Rodrigo Jiménez de Rada, Eb. von Toledo 72

Romaricus, A. von Remiremont 50,51

Rouen 52

Rudolf von Rheinfelden, Kg. 176

Rufinus 95

Sachsen 179

Salzburg 22, 23

Santiago de Compostela 178

St. Blasien 156-158

Saint-Denis $\quad 56,174,177-178$

St. Gallen 83

Saint-Gildas-de-Rhuys 50

Schmid, Karl 16, 154

Schmitt, Jean-Claude 24

Schramm, Percy Ernst 195

Sevilla 64, 68

Siegburg, Kloster 183

Sigibert I., Kg. $\quad$ 45, 47-48
Sigibert III., Kg. 146

Sigismund, Kg. $\quad 38,117,133$

Sigwin, Gf. 89

Silvester I., Pp. 44

Siricius, Pp. 129

Sisebut, Kg. $63,65-67,70$

Smith, Anthony D. 36

Sokrates 9-10

Solignac 57

Sozomenos 9-10

Spanien 179

Steinacker, Harold 116

Stock, Brian 20

Stratmann, Martina 134

Sturbius, Gf. $\quad 89$

Sulpicius Alexander 95

Suplicius Severus 26

Symmachus, Pp. 130,134

Tassilo III., Hz. 19

Thegan 174

Theoderich I., Kg. $\quad 80-81,117$

Theodor, B. von Mopsuestia 51

Theodor I., Pp. 126

Theodoret, B. von Cyrus 10,51

Theodosius II., Ks. 183

Theodrada 84

Theodulf, B. von Orléans 85

Theofilactus 86

Theudebert I., $\mathrm{Kg}$. 44, 52

Theuderich II., Kg. $\quad 46-52,53$

Theuderich III., Kg. 142

Theuderich IV., Kg. 146

Thier, Andreas 132

Thietmar von Merseburg 3

Tolbiac/Zülpich 43

Toledo $\quad 62-68,70-71,73,75$

Toulouse 119

Tours 99, 103

Turner, Cuthbert Hamilton 108, 115

Turpin, Eb. von Reims 175

Ubl, Karl 143

Udalrich, Gf. 83

Ulrich 157, 158

Unibert, Gf. 88

Urgell 179

Ussy 52

Valery 49 
Van Houts, Elisabeth 21-22

Vannes 47

Venantius Fortunatus $41,99,194$

Verbaal, Wim 25

Vergil 97, 98

Victorius von Aquitanien 50

Vigilius, Pp. 113, 130

Viktor III., B. von Chur 24, 83

Vouillé 119

Waifar, Hz. $\quad 88,89$

Waitz, Georg 132

Walahfrid Strabo, A. von Reichenau 83-85

Waldebert, A. von Luxeuil 53

Waldelenus 53

Wamba, Kg. 75

Warburg, Aby 195-197
Wenskus, Reinhard 36

Wilhelm, Sohn der Dhuoda 80-81

Wilhelm von Gellone, Gf. von Toulouse 89

Wilhelm von Malavalle 161-162, 169

Wilhelm von Malmesbury 179

Wilhelm X., Hz. von Aquitanien 162

Winter, Jay 15

Wissembourg 23

Witterich, Kg. $\quad 63,66,70$

Wood, Ian 36

Wollasch, Joachim 16

Yates, Frances 15,18

Zechiel-Eckes, Klaus 10, 108-109, 129

Zosimus I., Pp. 128

Zumthor, Paul 193 